http://dx.doi.org/10.7494/drill.2015.32.4.731

\title{
Igor Paliychuk*
}

\section{ELASTIC INTERACTION OF COUPLINGS AND PIPES WALLS IN TIGHT THREADED JOINTS}

\section{INTRODUCTION}

Box threaded connections of pipes are used during oil and gas recovery. The pipe and the coupling interact elastically by their tapered surfaces with radial tightness in the thread and the sealing. The strength and impermeability of the connection depends on this interaction.

The pipe and the coupling are thin-walled cylinders with the linear variable wall thickness. Their sections have varying lengthwise rigidity of walls and axial-symmetric bends. Therefore, their calculation differs from the Lame problem and from the calculation of the cylinders with the fixed wall thickness.

Physical and mathematical principles of the calculating parameters of elastic-strained state of all parts of pipe walls and coupling connections with the sealing box have been formulated in the article.

\section{ELASTIC STRAINS OF CYLINDER WALL WITH LINEAR VARIABLE THICKNESS}

The pipe and coupling have some sections (thread, sealing and groove, runout of thread, coupling center and barrel), which differ in functional and geometrical characteristics. The distributed pressure $P(x)$ works on each section inside and outside (Fig. 1). This can be the pressure of fluid or gas, fixed within the connection, or contact pressure on the seal and thread surfaces.

Adjacent sections serve as the support for each other in edge cross-sections, where the edge curvings are created depending on their size, loading and strains.

\footnotetext{
* Ivano-Frankivsk National Technical University of Oil and Gas, Ivano-Frankivsk, Ukraine
} 


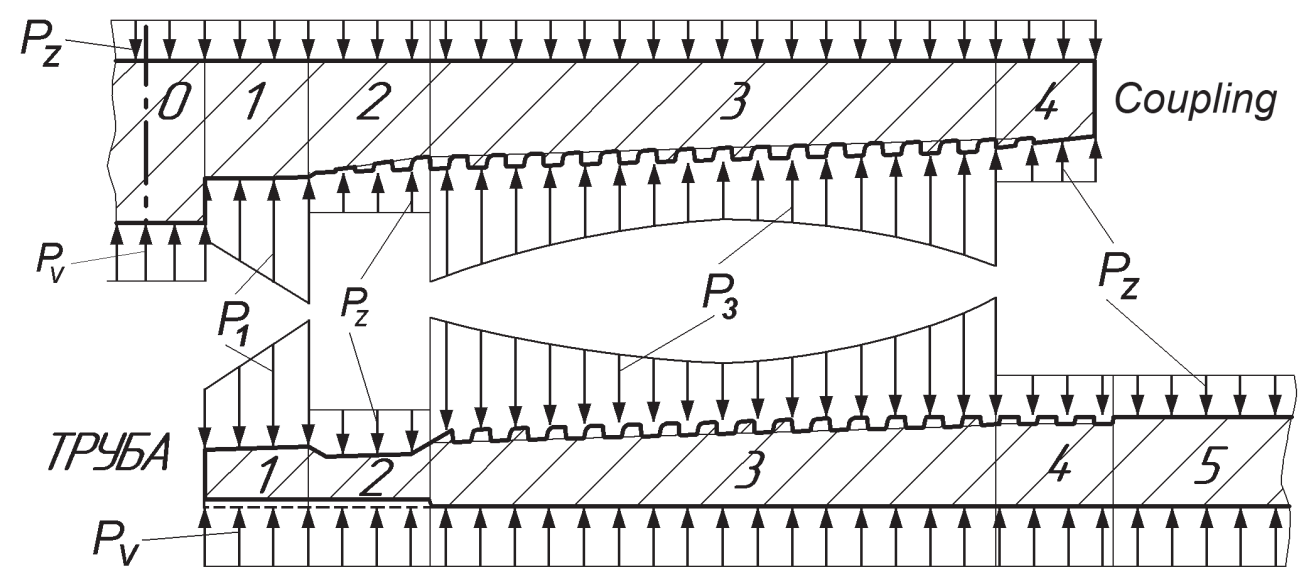

Fig. 1. Scheme of interaction of couplings and pipes plots in the joint under pressures $\left(P_{V}\right.$ as internal and $P_{Z}$ as external operating pressures; contact pressures are $P_{1}$ on seal and $P_{3}$ on thread sections)

Axial-symmetric strains of each sections wall are described by differential equation $[1,3]$.

$$
\frac{1}{x} \cdot \frac{d^{2}}{d x^{2}}\left(x^{3} \frac{d^{2} w}{d x^{2}}\right)+\rho^{4} w=\rho^{4} \frac{R^{2}}{E \cdot \operatorname{tg} \phi} \cdot \frac{P(x)}{x}
$$

where:

$E$ and $\mu-$ the modulus of elasticity and Poisson's ratio,

$R$ - radius of the middle surface,

$w$ - radial shift (change of radius) of the wall in cross-section $x$, notations are introduced.

$$
\rho=\sqrt[4]{\frac{12\left(1-\mu^{2}\right)}{R^{2}(\operatorname{tg} \phi)^{2}}}, y=2 \rho \sqrt{x}, D=\frac{E \cdot(x \cdot \operatorname{tg} \phi)^{3}}{12 \cdot\left(1-\mu^{2}\right)}
$$

The solution $w(x)$ of the homogeneous equation (2) with a right part, equal to null, contains a linear combination of derivatives from zero order of Bessel functions $[1,3]$.

$$
w=\frac{C_{1} \operatorname{ber}^{\prime} y+C_{2} \operatorname{bei}^{\prime} y+C_{3} \operatorname{ker}^{\prime} y+C_{4} \operatorname{kei}^{\prime} y}{\sqrt{x}}
$$

where:

' (prime) - marks differentiation by argument $y$,

$C_{1}, \ldots, C_{4}-$ constants of integration, which are determined from edge conditions,

ber $y$, bei $y$ - according to the real and imaginary parts of Bessel function of the first kind,

ker $y$, kei $y$ - the same parts of the modified Bessel function of the second kind.

These functions are in programming systems MATLAB or MAPLE. This allows their numeral calculation. 
According to moment theory of rotational shells [1] solution $w$ (4) of the equation (2) enables to define all characteristics, which determine the elastic-strained state of a wall in cross-sections with a coordinate $x$, in particular, in edge cross-sections.

$$
v=\frac{d w}{d x}, M=D \frac{d^{2} w}{d x^{2}}, N=\frac{d M}{d x}
$$

where:

$$
\begin{aligned}
& v \text { - angular deflection (slope) of a wall, } \\
& M \text { - bending moment, } \\
& N \text { - transverse (shearing) force. }
\end{aligned}
$$

The expressions of derivatives (5) are defined in detail [1, 3] also contain the same functions as (4).

Parameters $w, v, M, N(4-5)$ in a cylinder wall are axially symmetrical, that is, they are circular distributed and radially directed. They are obtained from the solution of the homogeneous equation; therefore they describe the strained walls state only from parameters, which act on a section in edge cross-sections from the side of the adjoining sections.

\section{CONTACT PRESSURE AND STRAINS OF WALLS OF TWO CYLINDERS, CONNECTED WITH TIGHTNESS}

Walls of the pipe and coupling, which are connected with normalized tightness, get axially symmetrical (ring-type and axled flexing) strains. They do not exceed moment theory application and satisfy the equation (2). Therefore, contact pressure, which arises in the connection owing to these strains also satisfies this equation; it is axially symmetrical, but varying lengthwise $[2,3]$.

The partial solution of differential equation (2) is offered for the effects account of the distributed pressure $P(x)$. It forms a right part of (2) and looks like:

$$
w^{*}=\frac{R^{2}}{E \cdot \operatorname{tg} \phi} \cdot \frac{P(x)}{x}
$$

At the same time the solution must satisfy the equation (2) on the whole, and it is possible only when the first component of this equation disappears.

$$
\frac{1}{x} \cdot \frac{d^{2}}{d x^{2}}\left(x^{3} \frac{d^{2}}{d x^{2}}\left(\frac{P(x)}{x}\right)\right)=0
$$

The equation (7) is a condition for the determination of contact pressure $P(x)$, offered for the first time [3] by the moment theory of rotational shells. After integration (7) general expression is obtained:

$$
P(x)=K_{1} \cdot x+K_{2} \cdot x^{2}+K_{3}+K_{4} \cdot x \cdot \ln x
$$

where $K_{1}, \ldots, K_{4}$ - constants of integration determined from interacting conditions of contact sections in the connection. 
From (6) and (8) expression of the radial shifts of a wall under the influence of contact pressure is obtained:

$$
w^{*}=\frac{R^{2}}{E \cdot \operatorname{tg} \phi}\left(K_{1}+K_{2} \cdot x+K_{3} \cdot \frac{1}{x}+K_{4} \cdot \ln x\right)
$$

whence accordingly (5) are obtained:

$$
v^{*}=\frac{R^{2}}{E \cdot \operatorname{tg} \phi}\left(K_{2}-K_{3} \cdot \frac{1}{x^{2}}+K_{4} \cdot \frac{1}{x}\right), M^{*}=\frac{1}{\rho^{4}}\left(2 K_{3}-K_{4} \cdot x\right), N^{*}=-\frac{K_{4}}{\rho^{4}}
$$

Characteristics (9)-(10) - shift $w^{*}$, slope $v^{*}$, bending moment $M^{*}$ and transverse force $N^{*}$ in the wall are caused by pressures, which are in the connection. They are obtained from the partial solution of the equation (2) and thus describe the strains of the cylinder with free flanks.

The complete elasticly strained state of the section is calculated as the sum of the corresponding characteristics (4)-(5) from the edge supports on the adjoining sections and characteristics (9)-(10) from pressures, which act on the section in the connecting.

\section{PRINCIPLE OF EQUILIBRIUM OF EDGE CROSS-SECTIONS OF ADJOINING SECTIONS IN THE CONNECTION OF A PIPE AND A COUPLING}

Final (resultant) values of characteristics, which were formed in the pipe and coupling walls at their connection with tightness and under the influence of operating pressure are determined separately for each section in the given cross-sections by the sum of the corresponding components (4)-(5) and (9)-(10) according to the equations set

$$
\begin{cases}W(x)=w+w^{*}+w_{0}, & M(x)=M+M^{*}+M_{0} \\ V(x)=v+v^{*}+v_{0}, & N(x)=N+N^{*}\end{cases}
$$

where $w_{0}, v_{0}, M_{0}$ - accordingly a shift, a slope and a bending moment in the wall, which create by fixed fluid pressure $P_{0}$.

They are similar to the component with an index 3 in formulas (8)-(10).

The fixed coefficients $C_{i}, K_{i}(4)-(5),(9)-(10)$ remain unknown after calculation of set components (11). For their definition the physical mechanism of formation of wall curvings of sections is used - compatibility of their strains [1]: walls are non-separable (continuous) in edge cross-section, common for two sections. The principle of equilibrium of cross-sections follows from this: resultant characteristics $W, V, M, N$ each of two adjoining sections are equal in the common cross-section.

Indexes are applied: the first $i$ specifies the given section; second $j-$ an adjoining section, which supports $i$-section: $j=i \pm 1$. From the set (11) the combined equations are obtained according to the equilibrium condition of cross-sections, which are linear concerning the unknown coefficients. 


$$
\left\{\begin{array} { l l } 
{ W _ { i } ( x _ { i , j } ) = W _ { j } ( x _ { j , i } ) , } & { M _ { i } ( x _ { i , j } ) = M _ { j } ( x _ { j , i } ) } \\
{ V _ { i } ( x _ { i , j } ) = V _ { j } ( x _ { j , i } ) , } & { N _ { i } ( x _ { i , j } ) = N _ { j } ( x _ { j , i } ) }
\end{array} \rightarrow \left\{\begin{array}{l}
w_{i, j}+w_{i, j}^{*}-w_{j, i}-w_{j, i}^{*}=w_{0 j, i}-w_{0 i, j} \\
v_{i, j}+v_{i, j}^{*}-v_{j, i}-v_{j, i}^{*}=v_{0 j, i}-v_{0 i, j} \\
M_{i, j}+M_{i, j}^{*}-M_{j, i}-M_{j, i}^{*}=M_{0 j, i}-M_{0 i, j} \\
N_{i, j}+N_{i, j}^{*}-N_{j, i}-N_{j, i}^{*}=0
\end{array}\right.\right.
$$

From the set (12) 4 equations are made for each edge cross-section of the pipe and coupling. As a result each section of the connection, which had 4 unknown of coefficient $C_{i}$, gets 4 equations for their definition.

\section{EQUILIBRIUM PRINCIPLE OF CONTACT PRESSURES IN THE CONNECTION OF THE PIPE AND COUPLING WITH TIGHTNESS}

The contact pressure appears on contact surfaces (in seal, in thread) after screwing the pipe and coupling with tightness. Its value is enough to expand the coupling and simultaneously to compress the pipe to form the connection. The internal pressure acts from inside on a pipe and increase its radius. The external pressure operates on a coupling and diminishes its radius. Due to these pressures contact pressure increases. As a result in the connection the equilibrium between strains and contact pressure, which are created by screwing tightness and operating pressures is created.

The physical mechanism of formation of elastic connection with tightness is used to find unknown coefficients $K_{i}$. It lies in the fact that the mutual radial action of the walls against each other is equal in value and opposite in direction on the contact sections of the pipe and coupling. The equilibrium principle of contact preassures is formulated out of: the sum of values of these pressures is equal to zero in each cross-section along the contact section. The mathematical expression of this condition is as follows:

$$
P_{m}\left(x_{m}\right)+P_{t}\left(x_{t}\right)=0
$$

where:

$m$ and $t$ - indexes of parameter correspondence to the coupling and the pipe, $x_{m}$ and $x_{t}$ - coordinates of one cross-section of the connection, but within their own reference frames,

$P_{m}\left(x_{m}\right)$ and $P_{t}\left(x_{t}\right)$ - values of contact pressure in this cross-section separately for the coupling and the pipe.

These coordinates and functions were expressed through one variable $X$ in general system of co-ordinates with beginning in symmetry plane of the screwed connection (in the coupling center). Taylor's expansion into a power series on derivatives [2] is applied to the functions. The equation (13) got the form:

$$
\begin{aligned}
P_{m}(X)+P_{t}(X)= & \left\{P_{m C}+P_{t C}\right\}- \\
& -\left(X-X_{C}\right)\left\{P_{m C}^{\prime}-P_{t C}^{\prime}\right\}+\frac{1}{2}\left(X-X_{C}\right)^{2}\left\{P_{m C}^{\prime \prime}+P_{t C}^{\prime \prime}\right\}- \\
& -\frac{1}{6}\left(X-X_{C}\right)^{3}\left\{P_{m C}^{\prime \prime \prime}-P_{t C}^{\prime \prime \prime}\right\}+\frac{1}{24}\left(X-X_{C}\right)^{4}\left\{P_{m C}^{I V}+P_{t C}^{I V}\right\}=0
\end{aligned}
$$

where $C$-an index, which marks the parameter value in the middle cross-section of the section. 
The derivatives from pressure are obtained by the differentiation of the expression (8):

$$
\begin{array}{ll}
P^{\prime}(x)=K_{1}+2 x K_{2}+K_{4}(\ln x+1), & P^{\prime \prime \prime}(x)=-x^{-2} K_{4} \\
P^{\prime \prime}(x)=2 K_{2}+x^{-1} K_{4}, & P^{I V}(x)=2 x^{-3} K_{4}
\end{array}
$$

The equilibrium equation of the pressures (13) must be fulfilled on the whole length of the contact section irrespective of coordinate $X$. Therefore, condition (14) is fulfilled only when each expression in braces (14) is equal to zero. For this purpose expressions of derivatives (15) are used and the system of linear equations is obtained relative to coefficients $K_{i}$.

$$
\begin{gathered}
x_{m C} K_{1 m}+x_{m C}^{2} K_{2 m}+1 \cdot K_{3 m}+x_{m C} \ln x_{m C} \cdot K_{4 m}+x_{t C} K_{1 t}+x_{t C}^{2} K_{2 t}+1 \cdot K_{3 t}+x_{t C} \ln x_{t C} \cdot K_{4 t}=0 \\
1 \cdot K_{1 m}+2 x_{m C} K_{2 m}+0 \cdot K_{3 m}+\left(\ln x_{m C}+1\right) K_{4 m}-1 \cdot K_{1 t}-2 x_{t C} K_{2 t}-0 \cdot K_{3 t}-\left(\ln x_{t C}+1\right) K_{4 t}=0 \\
2 \cdot K_{2 m}+x_{m C}^{-1} K_{4 m}+2 \cdot K_{2 t}+x_{t C}^{-1} K_{4 t}=0 \\
-K_{4 m} \cdot x_{m C}^{-2}+K_{4 t} \cdot x_{t C}^{-2}=0
\end{gathered}
$$

Consequently, according to the condition of pressure equilibrium it is possible to form 4 equations from the necessary 8 for each contact section of the connection.

\section{BALANCE PRINCIPLE OF WALL STRAINS AND TIGHTNESS ON THE CONTACT SECTIONS OF THE CONNECTION}

When disconnected the radius of the contact surface of the pipe exceeds the radius of the coupling by the value of the radial tightness $\Delta$. When screwing the pipe comes into the coupling and elastically unclasps it, while the coupling compresses the pipe. There appears the tight contact between their surfaces.

The physical mechanism of tight connection formation is in the fact that the radial shift $W_{m}$ of the coupling wall cannot become exceed tension $\Delta$, diminished by the pipe contraction $W_{t}$. On the other side, for the contact to bee, the pipe wall cannot be radially narrower than tightness $\Delta$, diminished by the widening $W_{m}$ of the coupling.

The principle of strains and tightness balance is based on this: the sum of the wall shifts of coupling $W_{m}$ (positive) and pipe $W_{t}$ (negative) in the connection is equal to the radial tightness $\Delta$ in any cross-section of the contact section. The mathematical expression of this condition is as follows:

$$
W_{m}\left(x_{m}\right)-W_{t}\left(x_{t}\right)=\Delta
$$

where:

$$
\begin{aligned}
x_{m}, x_{t}- & \text { coordinates of the given cross-section within their own reference } \\
& \text { frames, }
\end{aligned}
$$

The equation (13) and (17) are mathematically analogous. Therefore the method developed in item 4 is completely used for the solution of the equation (17). Instead of functions $P(x)$ functions $W(x)$ of the wall characteristics (11) are used in (17). They were put in the condition (17), where components with identical degrees of $X$ are grouped: 


$$
\begin{aligned}
W_{m}\left(x_{m}\right)-W_{t}\left(x_{t}\right)= & \left\{W_{m C}-W_{m C}\right\}-\left(X-X_{C}\right)\left\{W_{m C}^{\prime}+W_{t C}^{\prime}\right\}+ \\
& +\frac{1}{2}\left(X-X_{C}\right)^{2} \cdot\left\{W_{m C}^{\prime \prime}-W_{t C}^{\prime \prime}\right\}- \\
& -\frac{1}{6}\left(X-X_{C}\right)^{3}\left\{W_{m C}^{\prime \prime \prime}+W_{t C}^{\prime \prime \prime}\right\}+\cdots=\Delta
\end{aligned}
$$

The first braces contain the sum of wall shifts of the coupling and the pipe in the middle of the section, which is equal to tension $\Delta$. The remaining braces in (18) are equal to zero; the derivatives from functions $W(x)$ are put into them, where from the equation system is obtained in the general view:

$$
\begin{gathered}
w_{m C}+w_{*_{m} C}-w_{t C}-w_{*_{t C}}=\Delta-w_{0 m C}+w_{0 t C} \\
w_{m C}^{\prime}+w_{*_{m} C}^{\prime}+w_{t C}^{\prime}+w_{*_{t C}}^{\prime}=-w_{0 m C}^{\prime}-w_{0 t C}^{\prime} \\
w_{m C}^{\prime \prime}+w_{*_{m C}}^{\prime \prime}-w_{t C}^{\prime \prime}-w_{*_{t C}}^{\prime \prime}=-w_{0 m C}^{\prime \prime}+w_{0 t C}^{\prime \prime} \\
w_{m C}^{\prime \prime \prime}+w_{*_{m}}^{\prime \prime \prime}+w_{t C}^{\prime \prime \prime}+w_{*_{t C}}^{\prime \prime \prime}=-w_{0 m C}^{\prime \prime \prime}-w_{0 t C}^{\prime \prime \prime}
\end{gathered}
$$

Components of the equations (19) and their derivatives are discovered from (4)-(5) and (9)-(10). System (19) gives 4 more necessary equations, which are linear to coefficients $K_{i}$, for each contact section of the connection.

The calculation of the Bessel functions, components of parameters (4)-(5) and (9)-(10) and their derivatives, the solving of equation systems (12), (16), (19) are programmed in MATLAB medium. Realized computer program allows to discover and graphically to show contact pressure and characteristics of the elasticly strained state of the threaded connection of couplings and pipes with the seal box.

\section{CONCLUSION}

In the threaded connection of a pipe and coupling with the sealing box there occurs a complicated set of strain and power characteristics of elastic interaction of sections. The moment theory of rotational shells with the linear variable wall thickness was used to calculate this and the general expression of contact pressure was discovered for the first time. To determine the parameters there have been formulated physical and mathematical principles of equilibrium of edge cross-sections of adjoining sections, equilibrium of contact pressures, balance of strains and tightness on contact sections. It allows to calculation of these parameters (radial shifts, slopes, bending moments, transverse forces) of the elastic-strained state for walls of all connection sections under the influence of internal and external pressures.

\section{REFERENCES}

[1] Flugge W.: Statik und Dynamik der Schalen. Berlin: Springer-Verlag, 1957.

[2] Kryzhanivskyi Ye.I., Paliychuk I.I.: Metod rozrakhunku kontaktnoho tysku dlya mufty $i$ truby, z'yednanykh z natyahom. Naftohazova enerhetyka, vol. 1, no. 6, 2008, pp. 78-82.

[3] Paliychuk I.I.: Pruzhna vzayemodiya z'yednanykh z natyahom tsylindriv zi zminnoyu tovshchynoyu stinky. Rozvidka ta rozrobka naftovykh i gazovykh rodovyshch, vol. 2, no. 19, 2006, pp. 59-63. 